\title{
Study on Classification of Image Group
}

\author{
Un-Son Jong ${ }^{1}$, Song Won Kim ${ }^{2}$, Hye-Gyong Jang ${ }^{3}$, Myong-Chol $\mathrm{Ri}^{4}$, Yong Kim ${ }^{5}$, Song-Il $\mathrm{Ri}^{6}$ \\ ${ }^{1,2}$ Department of Physics, Kim Hyong Jik Normal University, Pyongyang, D. P. R. of Korea \\ ${ }^{3,5,6}$ Department of Mathematics, University of Science, Pyongyang, D. P. R. of Korea
}

${ }^{4}$ Institute of Physics Academy of Sciences, Pyongyang, Democratic People Republic of Korea Institute of Physics Chinese Academy of Sciences, Beijing, 100190, China

\begin{abstract}
Image of the irrep(irreducible representation) of the space group has the important significance in the study of the spontaneous symmetry breaking arising in the crystal. In this paper we defined the characteristic quantity of I-Group.And using it we established the procedure for finding the equivalent I-Group. We classified the irreps of the star channel group of the wave vector onto 142 image groups.
\end{abstract}

Keywords: representation; spacegroup; symmetry breaking; image

\section{Introduction}

An irrep of a space group $G$ maps the elements $g \in G$ onto matrices $D(g)$.

The set of distinct matrices is called the image of the irrep. Gufan [1] defined I-Group the and emphasized the role of it in the structural phase transition research and suggested the method for finding the IRBI (Integral Rational Basis of Invariant) and classified irreps corresponding 80 different Lifshitz stars onto 106 L-Groups.

Stokes [2] formed 4777 irreps corresponding 80 different Lifshitz stars using the irreducible representation table of ref [3] and classified onto 132 inequivalent images.

Such difference is related to the choice of the origin at the coordinate system and the method of forming the physically irreducible representation.
I-Group (Image-Group) are the set of distinct matrices onto which the space group elements are mapped by the irrep.

But there exist the I-Groups which are identical as the conjugate correct degree in the structural viewpoint between I-Groups of which the dimension of the representation's matrices and the order of the I-Group are the same. Since they are equivalent in the structural viewpoint, but the types of the matrices are different they were classified onto the distinct I-Groups.

For example, Let's Consider the distinct I-Gs( $D 32 \mu$, $D 32 \tau, D 32 \theta$ ) of which the dimension is 4 and the order is 32 .

The matrix types of each I-Groups are same as following.(Table 1,2,3)

Where $\sigma=\exp (i \pi / 4), \bar{\sigma}=\exp (-i \pi / 4)$.

Table 1: $D 32 \mu\left(D_{4}^{4}(16 / 1 \oplus 2)\right)$

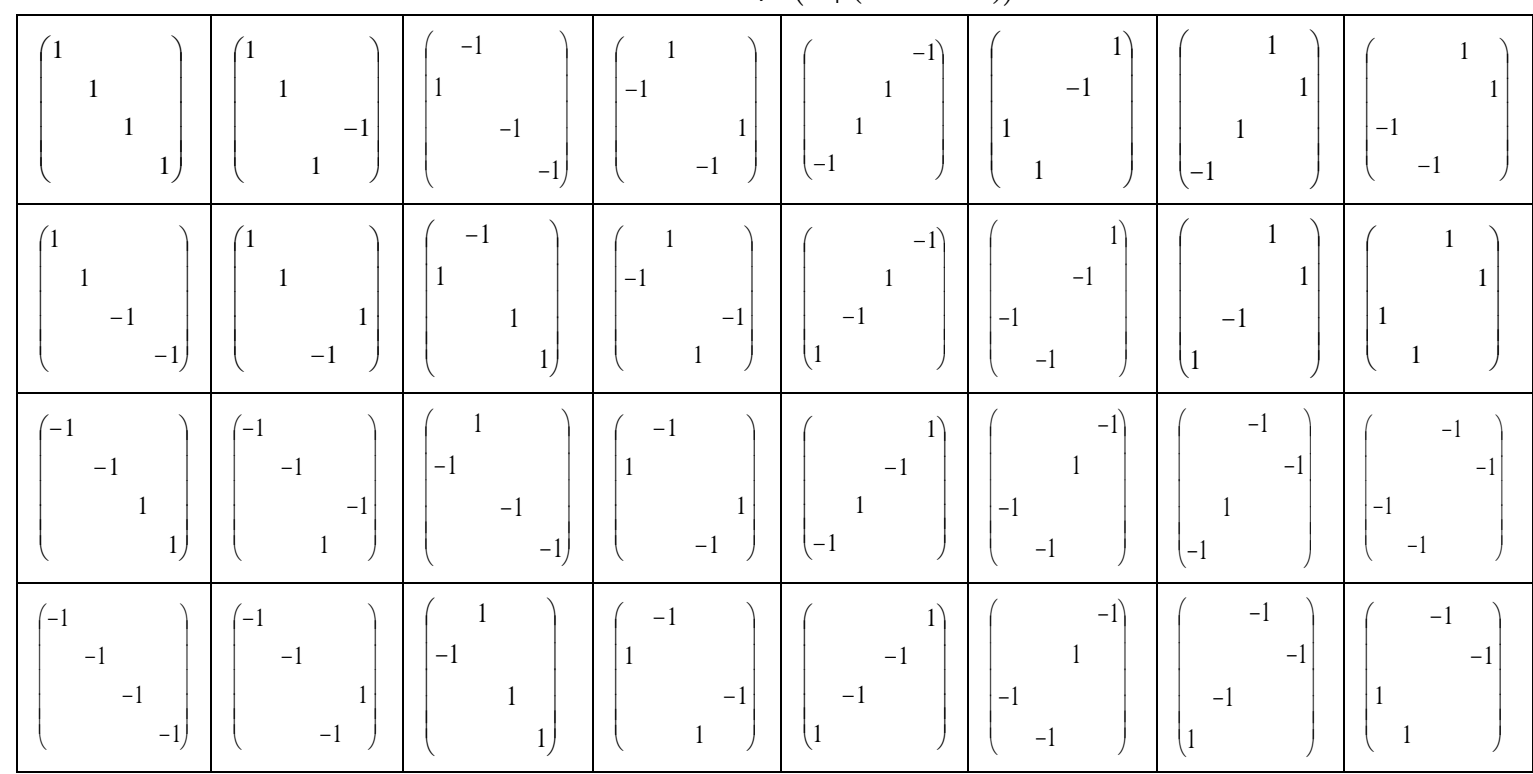

Volume 5 Issue 6, June 2016 www.ijsr.net 
International Journal of Science and Research (IJSR)

ISSN (Online): 2319-7064

Index Copernicus Value (2013): 6.14 | Impact Factor (2015): 6.391

Table 2: $D 32 \tau\left(C_{4 h}^{6}(12 / 1 \oplus 4)\right)$

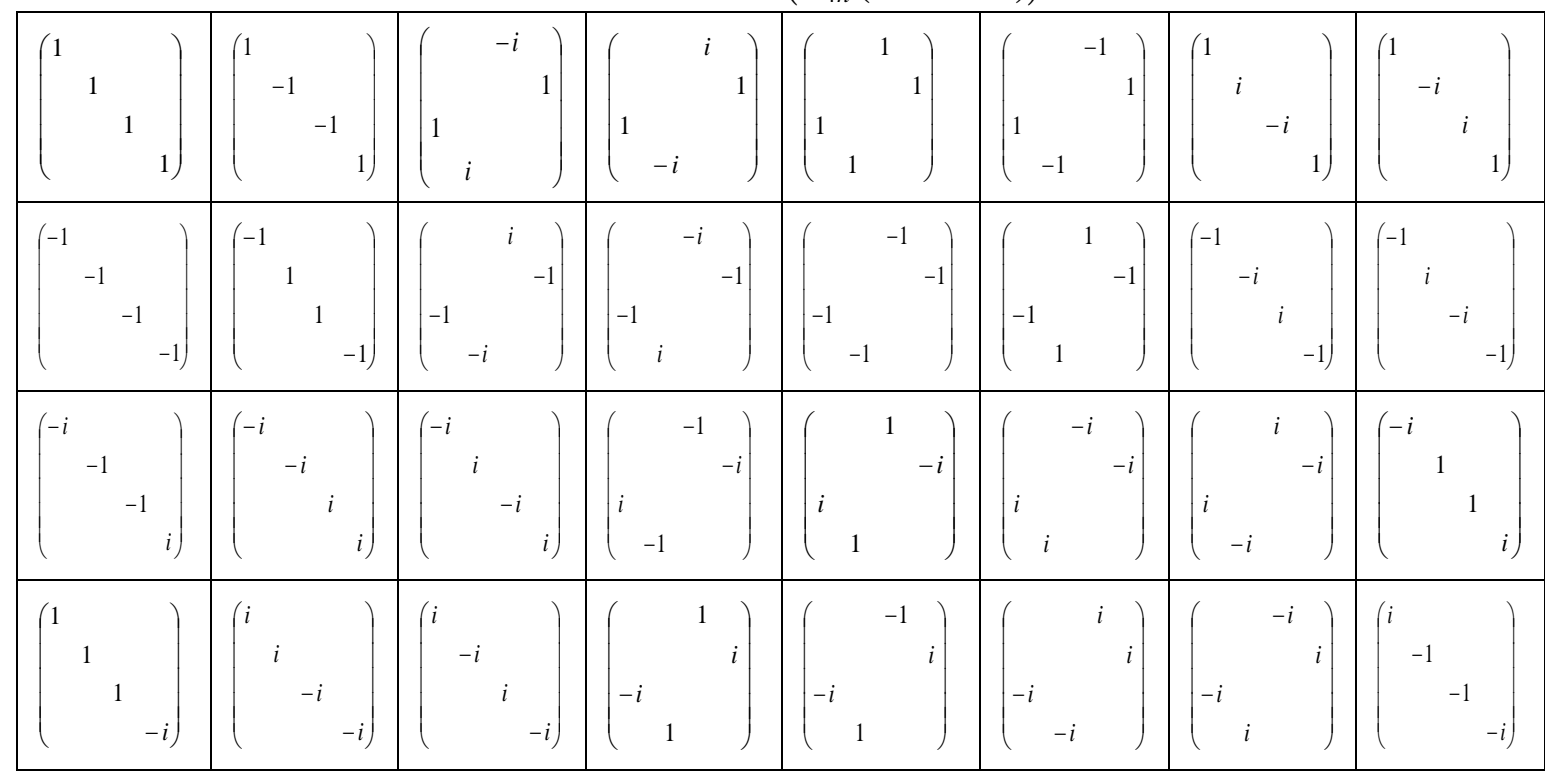

Table 3: $D 32 \theta\left(D_{2 d}^{12}(12 / 1 \times 2)\right)$

\begin{tabular}{|c|c|c|c|c|c|c|c|}
\hline$\left(\begin{array}{llll}1 & & & \\
& 1 & & \\
& & 1 & \\
& & & \\
& & & 1\end{array}\right)$ & $\left(\begin{array}{llll}1 & & & \\
& -i & & \\
& & 1 & \\
& & & \\
& & & i\end{array}\right)$ & $\left(\begin{array}{llll}1 & & & \\
& -1 & & \\
& & 1 & \\
& & & \\
& & & -1\end{array}\right)$ & $\left(\begin{array}{llll}1 & & & \\
& i & & \\
& & 1 & \\
& & & -i\end{array}\right)$ & $\left(\begin{array}{llll}-1 & & & \\
& -1 & & \\
& & -1 & \\
& & & -1\end{array}\right)$ & $\left(\begin{array}{llll}-1 & & & \\
& i & & \\
& & -1 & \\
& & & -i\end{array}\right)$ & $\left(\begin{array}{cccc}-1 & & & \\
& 1 & & \\
& & -1 & \\
& & & 1\end{array}\right)$ & $\left(\begin{array}{llll}-1 & & & \\
& -i & & \\
& & -1 & \\
& & & i\end{array}\right)$ \\
\hline$\left(\begin{array}{llll}-i & & & \\
& -i & & \\
& & i & \\
& & & \\
& & & i\end{array}\right)$ & $\left(\begin{array}{llll}-i & & & \\
& -1 & & \\
& & i & \\
& & & -1\end{array}\right)$ & $\left(\begin{array}{llll}-i & & & \\
& i & & \\
& & i & \\
& & & \\
& & & -i\end{array}\right)$ & $\left(\begin{array}{cccc}-i & & & \\
& 1 & & \\
& & i & \\
& & & 1\end{array}\right)$ & $\left(\begin{array}{llll}i & & & \\
& i & & \\
& & -i & \\
& & & -i\end{array}\right)$ & $\left(\begin{array}{llll}i & & & \\
& 1 & & \\
& & -i & \\
& & & 1\end{array}\right)$ & $\left(\begin{array}{llll}i & & & \\
& -i & & \\
& & -i & \\
& & & i\end{array}\right)$ & $\left(\begin{array}{llll}i & & & \\
& -1 & & \\
& & -i & \\
& & & -1\end{array}\right)$ \\
\hline$\left(\begin{array}{rrrr} & \overrightarrow{i \sigma} & & \\
-i \sigma & & & \\
& & & i \sigma \\
& & & i \sigma\end{array}\right)$ & $\left(\begin{array}{cccc} & \overrightarrow{ } & & \\
\overrightarrow{ }_{-i \sigma} & & & \\
& & & \\
& & & -\sigma \\
& & i \sigma & \end{array}\right)$ & 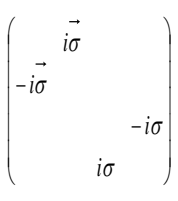 & $\left(\begin{array}{cccc} & \vec{\sigma} & & \\
-\overrightarrow{i \sigma} & & \\
& & & \\
& & & \\
& & i \sigma & \end{array}\right)$ & 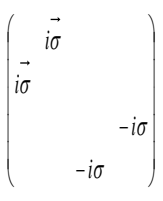 & $\left(\begin{array}{cccc} & \vec{\sigma} & & \\
\overrightarrow{i \sigma} & & & \\
& & & \\
& & & \\
& & -i \sigma & \end{array}\right)$ & $\left(\begin{array}{cccc} & -\overrightarrow{i \sigma} & & \\
\overrightarrow{i \sigma} & & & \\
& & & i \sigma \\
& & -i \sigma & \end{array}\right)$ & $\left(\begin{array}{cccc} & & -\vec{\sigma} & \\
\overrightarrow{i \sigma} & & \\
& & & \\
& & & -\sigma \\
& & -i \sigma & \end{array}\right)$ \\
\hline$\left(\begin{array}{cccc} & -\vec{\sigma} & & \\
-\vec{\sigma} & & \\
& & & -i \sigma \\
& & & \\
& & -i \sigma & \end{array}\right)$ & 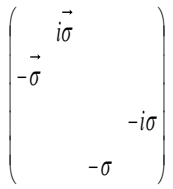 & $\left(\begin{array}{cccc} & \vec{\sigma} & & \\
-\vec{\sigma} & & & \\
& & & \sigma \\
& & & \\
& & -\sigma & \end{array}\right)$ & $\left(\begin{array}{cccc} & -\overrightarrow{i \sigma} & & \\
-\vec{\sigma} & & & \\
& & & i \sigma \\
& & & \\
& & \sigma & \end{array}\right)$ & $\left(\begin{array}{cccc} & \vec{\sigma} & & \\
\vec{\sigma} & & & \\
& & & \sigma \\
& & & \\
& & \sigma & \end{array}\right)$ & $\left(\begin{array}{llll} & -i \vec{\sigma} & & \\
\vec{\sigma} & & & \\
& & & i \sigma \\
& & & \\
& & \sigma & \end{array}\right)$ & $\left(\begin{array}{llll} & -\vec{\sigma} & & \\
\vec{\sigma} & & \\
& & & -\sigma \\
& & & \\
& & \sigma & \end{array}\right)$ & $\left(\begin{array}{cccc} & \overrightarrow{i \sigma} & & \\
\vec{\sigma} & & & \\
& & & -i \sigma \\
& & & \\
& & \sigma & \end{array}\right)$ \\
\hline
\end{tabular}

Rep (representation) $D_{4}^{4}(16 / 1 \oplus 2)$ is the direct sum of the first rep $T_{1}$ and the second rep $T_{2}$ corresponding Lifshitz star $\vec{K}_{16}$ of the space group $P 4_{1} 2_{1} 2\left(D_{4}^{4}\right)$.

Rep $C_{4 h}^{6}(12 / 1 \oplus 4)$ is the direct sum of the first rep $T_{1}$ and the fourth rep $T_{4}$ corresponding Lifshitz star $\vec{K}_{12}$ of the space group $I 4_{1} / a\left(C_{4 h}^{6}\right)$.
Rep $D_{2 d}^{12}(12 / 1 \times 2)$ is the direct sum of the twice first rep $T_{1}$ corresponding Lifshitz star $\vec{K}_{12}$ of the space group $I \overline{4} 2 d\left(D_{2 d}^{12}\right)$.

Since the types of the above representation matrices are distinct, they were classified onto the distinct I-Groups $D 32 \tau, D 32 \mu, D 32 \theta$.

By the following unitary transformation $D 32 \mu(g)=U_{1} D 32 \tau(g) U_{1}^{-1}$

\section{Volume 5 Issue 6, June 2016} www.ijsr.net 


\section{International Journal of Science and Research (IJSR)}

ISSN (Online): 2319-7064

Index Copernicus Value (2013): 6.14 | Impact Factor (2015): 6.391

$$
D 32 \mu(g)=U_{2} D 32 \theta(g) U_{2}^{-1}
$$

The matrices of $D 32 \tau, D 32 \theta$ coincide with the type of matrix of $D 32 \mu$ and they are become an I-Group. Where the unitary transformation matrices $U_{1}$ and $U_{2}$ are the same as

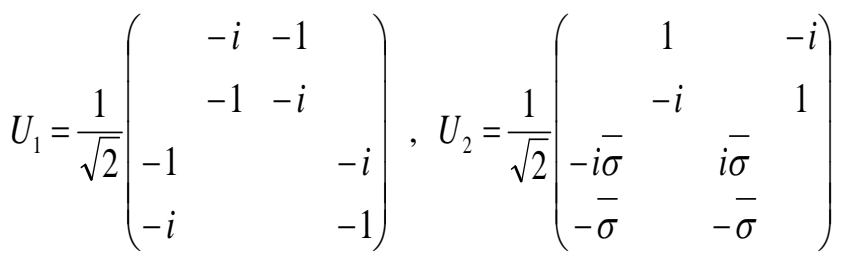

It is difficult to find the unitary transformation matrices coinciding the I-Groups of which the dimension and the order is the same. There is no practical method to find the such unitary transformation matrices.

Furthermore, it is very difficult problem to find the unitary transformation matrices coinciding with the equivalent I-Groups of which the dimension is 12 and the order is 1536.

It needs the new method to find the equivalent I-Group.

Otherwise the different physical phenomena arising in the solid crystals are related to not only all the starlines of the star of the wave vector but also some starlines of it.

In the [7] they found the irrep of the star channel group of the wave vector and suggested a three components order parameter model to describe the successive phase transitions due to condensation of several soft modes.

There must be established the procedure for classifying I-Group to find correctly the I-Groups of the irrep of the star channel group that is the important mathematical tool in the structural phase transition research of the crystal.

\section{The characteristic quantity of I-Group}

\section{1) The trace of I-Group matrix}

Denote the trace of the I-Group matrix $M(g)$ by $\chi(g)$.This is the sum of the diagonal elements of matrix in the arbitrary base.[5]

$$
\chi(g)=\sum_{i=1}^{s} M_{i i}(g)
$$

Where $g$ is the number of I-Group matrices and it equals the order of I-G.

$\mathrm{S}$ is the dimension of the matrix.

The traces of I-Group matrices are invariant on the resemblance transition.

In fact, if

$$
M^{\prime}(g)=U M(g) U^{-1}
$$

Then

$$
\begin{aligned}
& \chi^{\prime}(g)=\sum_{i} M_{i i}^{\prime}(g)=\sum_{i j k} U_{i j} M_{j k}(g) U_{k i}^{-1}= \\
& =\sum_{i j k} M_{j k}(g)\left(U^{-1} U\right)_{k j}=\sum_{j} M_{j j}(g)=\chi(g)
\end{aligned}
$$

Since the trace of I-Group matrix is invariant on the resemblance transition, the trace of the equivalent I-Group's matrix must be equal.

\section{2) The order of I-Group matrix}

One property of the representation matrix is that the unit matrix is get by multiplating itself limitedness

$$
M^{n}(g)=E
$$

This $\mathrm{n}$ is called the order of the I-Group matrix

The order of I-Group matrices are invariant on the resemblance transition.

$$
\begin{aligned}
& M^{\prime}(g)=U M(g) U^{-1} \\
& M^{\prime n}(g)=\left(U M(g) U^{-1}\right)^{n}=U M(g) U^{-1} \cdot U M(g) U^{-1} \cdots \\
& \cdots U M(g) U^{-1}=U M^{n}(g) U^{-1}=U E U^{-1}=E
\end{aligned}
$$

As the order of I-Group's matrices is invariant on the resemblance transition, the order of the equivalent I-Group's matrices must be equal.

Though the trace and the order of matrix of I-G are the same, the number of matrices corresponding them can be different. It is related to the kernel of irreducible representation.

\section{3) The dimension of subspace of I-Group}

By the Landau theory [6], the change $\Delta \rho(\vec{r})$ of the dencity function $\rho(\vec{r})$ which find in the spontaneous symmetry breaking phase transition of the crystal can be expanded by the basis functions $\varphi_{m}(\vec{r})$ of the some irreducible representation $T^{v}$ of the symmetry group $G_{0}$.

$$
\Delta \rho(\vec{r})=\sum_{m} \vec{\eta}_{m} \varphi_{m}(\vec{r})
$$

The coefficients $\vec{\eta}_{m}$ formed an $m$-dimensional vector $\vec{\eta}$ called the order parameter. The symmetry group $G_{0}$ obtained from the distortion consists of all element $g \in G_{0}$ which leave the distortion invariant. Such a group is called an isotropy subgroup of $G_{0}$. 


\section{International Journal of Science and Research (IJSR) \\ ISSN (Online): 2319-7064}

Index Copernicus Value (2013): 6.14 | Impact Factor (2015): 6.391

Because $\mathrm{I}-\mathrm{G}$ acts as the abstracting point group in the space $\varepsilon_{n}$, the space $\varepsilon_{n}$ is disjointed by the orbits, which is the set of the different vectors that are get operating all the elements of the group to the given vector $\vec{\eta}$.

The dimension of the invariant subspace to the subgroup $H_{\alpha}$ that leave invariant the nonzero subspace of the

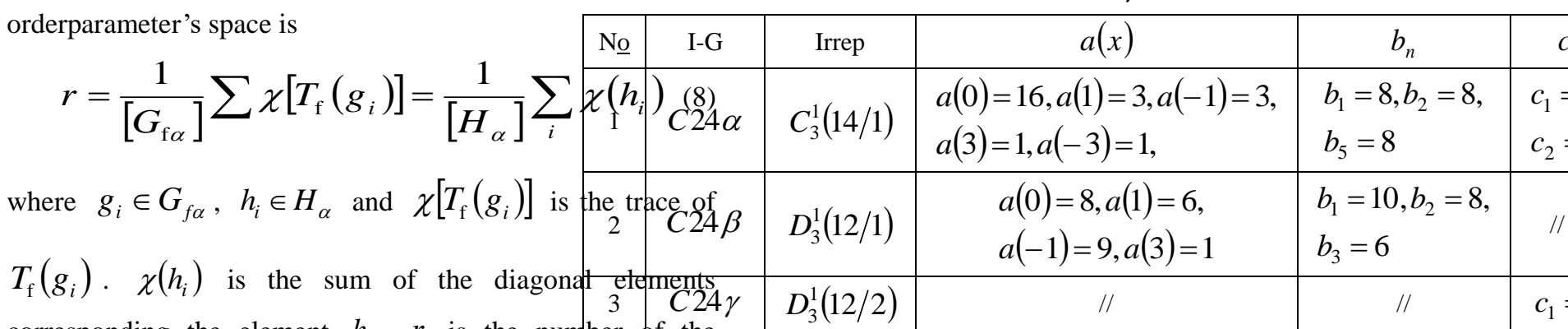

corresponding the element $h_{i}, r$ is the number of the independent part of the order parameter $\vec{\eta}$ that characterize the isotropy subgroup. Actually, the number of $H_{\alpha}$ of the equivalent I-Group must be equal.

\section{The condition for existence of the unitary matrix for finding the equivalent I-G}

We formulate the existence condition of the unitary transformation of the I-Group as following.

Given two I-Gs $I_{1}, I_{2}$

We denote their matrices and subgroups by $M_{1}(g)$, $M_{2}(g), H_{1 \alpha}, H_{2 \alpha}$ and the order by $n(M(g))$, the number of subgroups by $r\left(H_{\alpha}\right)$.

If the following relation stands between two I-Gs,

$$
\chi\left(M_{1}(g)\right)=\chi\left(M_{2}(g)\right)
$$$$
n\left(M_{1}(g)\right)=n\left(M_{2}(g)\right)
$$$$
r_{\alpha}\left(H_{1 \alpha}\right)=r_{\alpha}\left(H_{2 \alpha}\right)
$$

there exists surely the unitar's transformation matrix $U$ satisfied

$$
M_{2}(g)=U M_{1}(g) U^{+}
$$

and two I-Groups are equivalent.
For example, let's consider the I-Groups $C 24 \alpha, C 24 \beta$, $C 24 \gamma$ with the dimension 3 and the order 24. In the bellow table we presented the characteristic quantity of each the I-Groups.

Table 4: Characteristic quantity of $C 24 \alpha, C 24 \beta$,

In the irrep column is only indicated a representation of the space group corresponding to I-G. Where rep $C_{3}^{1}(14 / 1)$ is the first rep of the Lifshitz star $\vec{K}_{14}$ of the spacegroup $C_{3}^{1}$. $a(x)$ is the number of matrices having the trace of the matrix $x, b_{n}$ is the number of matrices having the order $\mathrm{n}$, $c_{r}$ is the number of $\mathrm{r}$ dimension subspace.

Table1 shows that the traces and the orders of $C 24 \beta$ and $C 24 \gamma$ are the identical, but the number of the subspace are not identical, the number of the subspace of $C 24 \alpha$ and $C 24 \beta$ are identical.

Because of the three conditions don't satisfy, the above I-Groups are classified the non-equivalent I-Groups.

For example, Let's consider 4 dimension I-Gs $D 48 \chi, D 48 \delta, D 48 \gamma, D 48 \beta, D 48 \alpha$. 


\section{International Journal of Science and Research (IJSR) \\ ISSN (Online): 2319-7064}

Index Copernicus Value (2013): 6.14 | Impact Factor (2015): 6.391

Table 5: Characteristic quantity of $D 48 \chi, D 48 \delta, D 48 \gamma, D 48 \beta, D 48 \alpha$

\begin{tabular}{|c|c|c|c|c|c|}
\hline № & I-G & Irrep & $a(x)$ & $b_{n}$ & $c_{r}$ \\
\hline 1 & $D 48 \chi$ & $O_{h}^{9}(10 / 3)$ & $\begin{array}{l}a\left(2 \varepsilon+2 \varepsilon^{2}\right)=2, a(0)=42, a(4)=1, \\
a\left(-2 \varepsilon-2 \varepsilon^{2}\right)=2, a(-4)=1\end{array}$ & $\begin{array}{l}b_{1}=24, b_{2}=2, b_{3}=8, \\
b_{5}=10, b_{11}=4\end{array}$ & $\begin{array}{c}c_{1}=4, \\
c_{2}=6\end{array}$ \\
\hline 2 & $D 48 \delta$ & $D_{6 h}^{4}(17 / 3)$ & $/ /$ & $/ /$ & $/ /$ \\
\hline 3 & $D 48 \gamma$ & $D_{6 h}^{4}(15 / 1)$ & $/ /$ & $/$ & $/ /$ \\
\hline 4 & $D 48 \beta$ & $D_{6 h}^{3}(17 / 3)$ & $/ /$ & $/ /$ & $/ /$ \\
\hline 5 & $D 48 \alpha$ & $D_{6 h}^{2}(15 / 1)$ & $/ /$ & $/$ & $/$ \\
\hline
\end{tabular}

Since the above I-Gs's characteristic quantities are the same, they are the equivariant I-Gs

There exist the unitar's matrices $U_{1} \sim U_{4}$ that satisfies the following expression

$$
\begin{aligned}
& D 48 \alpha(g)=U_{1} D 48 \chi(g) U_{1}^{+} \\
& D 48 \alpha(g)=U_{2} D 48 \delta(g) U_{2}^{+} \\
& D 48 \alpha(g)=U_{3} D 48 \gamma(g) U_{3}^{+} \\
& D 48 \alpha(g)=U_{4} D 48 \beta(g) U_{4}^{+}
\end{aligned}
$$

They are the same as follows.

$$
\begin{aligned}
& U_{1}=\frac{1}{\sqrt{2}}\left(\begin{array}{cccc}
1 & & 1 & \\
1 & & -1 & \\
& 1 & & 1 \\
& 1 & & -1
\end{array}\right) \quad U_{2}=\frac{1}{\sqrt{2}}\left(\begin{array}{cccc}
1 & & 1 & \\
1 & & -1 & \\
& 1 & & -1 \\
& -1 & & -1
\end{array}\right) \\
& U_{3}=\left(\begin{array}{llll}
1 & & & \\
& 1 & & \\
& & & i \\
& & i &
\end{array}\right) \quad U_{4}=\frac{1}{\sqrt{2}}\left(\begin{array}{cccc}
1 & & -i & \\
1 & & i & \\
& 1 & & i \\
& -1 & & i
\end{array}\right)
\end{aligned}
$$

\section{Procedure for the Classification of Image-Group.}

We establish the procedure for the classification of I-Group of the irreducible representation corresponding to the different Lifshitz's stars of the different spacegroup $G_{0}$ on the based the definition of the characteristic quantity of I-G and the condition for existence of unitary transformation.

The procedure consisted of two stages.

1)The stage finding I-Groups (1) (3))

(1) Find the homomorphic kernel $\operatorname{ker} T$ of the full irreducible representation of the spacegroup $G_{0}$.

(2) Find the factor group $G_{f}=G_{0} / \operatorname{ker} T$ by $\operatorname{ker} T$.

(3) Find the correct representation $T_{\mathrm{f}}$ of $G_{\mathrm{f}}$.

2)The stage classifying onto non-equivalent I-Groups.(4) (7))

(4) Find the traces, orders, the number of subspace among the I-Groups that have the same dimensions and orders.

(5) Decide the identical I-Group of the I-Groups that have the same characteristic quantity.

(6) Find the unitar's transformation matrices that coincide with the same I-Gs.

(7) Coincide with the equivalent I-Groups operating unitar's transformation $I_{1}(g)=U I_{2}(g) U^{+}$to all the matrices of I-Group.

Above classified I-Groups are the non-equivalent

We presented the number of the full irreducible representation of 230 space group corresponding to I-G in the table 6. 
International Journal of Science and Research (IJSR)

ISSN (Online): 2319-7064

Index Copernicus Value (2013): 6.14 | Impact Factor (2015): 6.391

Table 6: The number of the Irrep corresponding to I-G

\begin{tabular}{|c|c|c|c|c|c|c|c|c|}
\hline № & I-G & $\mathrm{n}$ & № & I-G & $\mathrm{n}$ & № & I-G & $\mathrm{n}$ \\
\hline 1 & $A 1 a$ & 230 & 25 & $D 24 b$ & 3 & 49 & $D 72 d$ & 12 \\
\hline 2 & $A 2 a$ & 1848 & 26 & $D 24 c$ & 5 & 50 & $D 96 a$ & 3 \\
\hline 3 & $B 3 a$ & 46 & 27 & $D 24 d$ & 3 & 51 & $D 96 b$ & 2 \\
\hline 4 & $B 4 a$ & 273 & 28 & $D 24 e$ & 20 & 52 & $D 96 c$ & 1 \\
\hline 5 & $B 6 a$ & 109 & 29 & $D 32 a$ & 2 & 53 & $D 96 d$ & 2 \\
\hline 6 & $B 6 b$ & 94 & 30 & $D 32 b$ & 51 & 54 & $D 128 a$ & 4 \\
\hline 7 & $B 8 a$ & 934 & 31 & $D 32 c$ & 29 & 55 & $D 144 a$ & 2 \\
\hline 8 & $B 8 b$ & 8 & 32 & $D 32 d$ & 3 & 56 & $D 192 a$ & 2 \\
\hline 9 & $B 12 a$ & 160 & 33 & $D 32 e$ & 8 & 57 & $D 192 b$ & 8 \\
\hline 10 & $B 12 b$ & 10 & 34 & $D 36 a$ & 4 & 58 & $D 192 c$ & 2 \\
\hline 11 & $B 16 a$ & 12 & 35 & $D 36 b$ & 16 & 59 & $D 384 a$ & 4 \\
\hline 12 & $B 24 a$ & 10 & 36 & $D 36 c$ & 18 & 60 & $E 48 a$ & 4 \\
\hline 13 & $C 12 a$ & 43 & 37 & $D 48 a$ & 2 & 61 & $E 48 b$ & 9 \\
\hline 14 & $C 24 a$ & 73 & 38 & $D 48 b$ & 2 & 62 & $E 48 c$ & 2 \\
\hline 15 & $C 24 b$ & 85 & 39 & $D 48 c$ & 2 & 63 & $E 96 a$ & 2 \\
\hline 16 & $C 24 c$ & 85 & 40 & $D 48 d$ & 1 & 64 & $E 96 b$ & 4 \\
\hline 17 & $C 48 a$ & 208 & 41 & $D 48 e$ & 5 & 65 & $E 96 c$ & 6 \\
\hline 18 & $D 8 a$ & 16 & 42 & $D 64 a$ & 4 & 66 & $E 96 d$ & 12 \\
\hline 19 & $D 12 a$ & 15 & 43 & $D 64 b$ & 2 & 67 & E96e & 8 \\
\hline 20 & $D 16 a$ & 2 & 44 & $D 64 c$ & 46 & 68 & $E 96 f$ & 12 \\
\hline 21 & $D 16 b$ & 3 & 45 & $D 64 d$ & 4 & 69 & $E 96 g$ & 1 \\
\hline 22 & $D 16 c$ & 60 & 46 & $D 72 a$ & 1 & 70 & $E 96 h$ & 2 \\
\hline 23 & $D 18 a$ & 16 & 47 & $D 72 b$ & 4 & 71 & $E 96 i$ & 2 \\
\hline 24 & $D 24 a$ & 3 & 48 & $D 72 c$ & 4 & 72 & $E 96 j$ & 2 \\
\hline № & I-G & $\mathrm{n}$ & № & I-G & $\mathrm{n}$ & № & I-G & $\mathrm{n}$ \\
\hline 73 & $E 96 k$ & 2 & 93 & $F 32 b$ & 1 & 113 & $F 384 b$ & 1 \\
\hline 74 & $E 192 a$ & 6 & 94 & $F 64 a$ & 4 & 114 & $F 384 c$ & 1 \\
\hline 75 & $E 192 b$ & 1 & 95 & $F 64 b$ & 1 & 115 & $G 96 a$ & 1 \\
\hline 76 & $E 192 c$ & 4 & 96 & $F 64 c$ & 1 & 116 & $G 192 a$ & 1 \\
\hline 77 & $E 192 d$ & 14 & 97 & $F 72 a$ & 2 & 117 & $G 192 b$ & 1 \\
\hline 78 & E192e & 14 & 98 & $F 72 b$ & 1 & 118 & G384a & 1 \\
\hline 79 & $E 192 f$ & 9 & 99 & $F 96 a$ & 3 & 119 & $G 384 b$ & 1 \\
\hline 80 & $E 192 g$ & 2 & 100 & $F 96 b$ & 1 & 120 & $G 384 c$ & 1 \\
\hline 81 & $E 192 h$ & 4 & 101 & $F 96 c$ & 1 & 121 & $G 768 a$ & 1 \\
\hline 82 & $E 192 i$ & 2 & 102 & $F 96 d$ & 1 & 122 & $G 768 b$ & 2 \\
\hline 83 & $E 192 j$ & 2 & 103 & $F 128 a$ & 1 & 123 & $G 768 c$ & 1 \\
\hline 84 & $E 384 a$ & 2 & 104 & $F 128 b$ & 1 & 124 & G768d & 1 \\
\hline
\end{tabular}

Volume 5 Issue 6, June 2016 www.ijsr.net

Licensed Under Creative Commons Attribution CC BY 
International Journal of Science and Research (IJSR)

ISSN (Online): 2319-7064

Index Copernicus Value (2013): 6.14 | Impact Factor (2015): 6.391

\begin{tabular}{|c|c|c|c|c|c|c|c|c|}
\hline 85 & $E 384 b$ & 2 & 105 & $F 144 a$ & 2 & 125 & $G 1536 a$ & 1 \\
\hline 86 & $E 384 c$ & 8 & 106 & $F 192 a$ & 4 & 126 & $G 1536 b$ & 2 \\
\hline 87 & $E 384 d$ & 2 & 107 & $F 192 b$ & 2 & 127 & $G 1536 c$ & 1 \\
\hline 88 & $E 768 a$ & 4 & 108 & $F 192 c$ & 1 & 128 & $G 1536 d$ & 1 \\
\hline 89 & $E 768 b$ & 4 & 109 & $F 192 d$ & 1 & 129 & $H 192 a$ & 1 \\
\hline 90 & $E 768 c$ & 2 & 110 & $F 192 e$ & 1 & 130 & $H 384 a$ & 1 \\
\hline 91 & $E 1536 a$ & 4 & 111 & $F 192 f$ & 1 & 131 & $H 384 b$ & 1 \\
\hline 92 & $F 32 a$ & 1 & 112 & $F 384 a$ & 2 & 132 & $K 1536 a$ & 1 \\
\hline
\end{tabular}

In thice table the first letter of the $\mathrm{I}-\mathrm{G}$ denotes the dimension of the matrices $(A=1, B=2, C=3, D=4, E=6, F=8, G=12$, $\mathrm{H}=16, \mathrm{~K}=24)$ and the number denotes the order of the Image-Group (number of distinct matrices) the final letter is arbitraliy chosen to distinguish images of the same dimension and order.

Concretely, 4777 irreps corresponding to 80 Lifshitz's stars Of 230 space groups were classified onto 132 I-Gs; 1 dimensional 2078 irreps were classified onto 2 I-Gs, 2 dimensional 1608 irreps were classified onto 10 I-Gs, 3 dimensional 494 irreps were classified onto 5 I-Gs, 4 dimensional 387 irreps were classified onto 42 I-Gs, 6 dimensional 154 irreps were classified onto 32 I-Gs, 8 dimensional 35 irreps were classified onto $23 \mathrm{I}-\mathrm{Gs}, 12$ dimensional 17 irreps were classified onto 14 I-Gs, 16 dimensional 3 irreps were classified onto 3 I-Gs, 24 dimensional one irrep was classified onto one I-G.

There are I-Groups of which the dimension is 12 and the order is 1536 , such as G1536b of the I-Groups to find the equivalent I-Groups

It is very difficult to find the unitary transformation matrix with large dimention and ordre by the experiential method.
So, the new method classifying I-Group is the most successful and correct

All the 8460 irreps of 2811 star channel group corresponding to 80 Lifshitz's stars of 230 space groups were classified onto 142 I-Gs by the above procedure. (Table 7)

1 dimensional 3965 irreps were classified onto 2 I-Gs, 2 dimensional 3 884irreps were classified onto 11 I-Gs, 3 dimensional 582 irreps were classified onto 5 I-Gs, 4 dimensional 575 irreps were classified onto 47 I-Gs, 6 dimensional 154 irreps were classified onto 32 I-Gs, 8 dimensional 50 irreps were classified onto 27 I-Gs, 12 dimensional 17 irreps were classified onto $14 \mathrm{I}-\mathrm{Gs}, 16$ dimensional 3 irreps were classified onto 3 I-Gs, 24 dimensional one irrep was classified onto one I-G.

The irreps of the star channel group of the wave vector was classified onto more 10 I-Groups than the full irreducible representations of the space group

That is 2 dimensional I-Group B4b, 4 dimensional I-Groups D16d, D32f, D64e, D128b, D128c, 8 dimensional I-Groups F64d, F256a, F256b, F256c.

Table 7: The number of the star channel group's irrep corresponding to I-G

\begin{tabular}{|c|c|c|c|c|c|c|c|c|}
\hline № & I-G & $\mathrm{n}$ & № & I-G & $\mathrm{n}$ & № & I-G & n \\
\hline 1 & A1a & 230 & 31 & D32a & 2 & 61 & D144a & 2 \\
\hline 2 & A2a & 3735 & 32 & D32b & 60 & 62 & D192a & 2 \\
\hline 3 & B3a & 46 & 33 & D32c & 60 & 63 & D192b & 8 \\
\hline 4 & B4a & 392 & 34 & D32d & 9 & 64 & D192c & 2 \\
\hline 5 & B4b & 6 & 35 & D32e & 8 & 65 & D384a & 4 \\
\hline 6 & B6a & 109 & 36 & D32f & 6 & 66 & E48a & 4 \\
\hline 7 & B6b & 114 & 37 & D36a & 4 & 67 & E48b & 9 \\
\hline 8 & B8a & 1973 & 38 & D36b & 16 & 68 & E48c & 2 \\
\hline 9 & B8b & 8 & 39 & D36c & 18 & 69 & E96a & 2 \\
\hline 10 & B12a & 1188 & 40 & D48a & 2 & 70 & E96b & 4 \\
\hline 11 & B12b & 14 & 41 & D48b & 2 & 71 & E96c & 6 \\
\hline 12 & B16a & 24 & 42 & D48c & 2 & 72 & E96d & 12 \\
\hline
\end{tabular}

Volume 5 Issue 6, June 2016 www.ijsr.net

Licensed Under Creative Commons Attribution CC BY 
International Journal of Science and Research (IJSR)

ISSN (Online): 2319-7064

Index Copernicus Value (2013): 6.14 | Impact Factor (2015): 6.391

\begin{tabular}{|c|c|c|c|c|c|c|c|c|}
\hline 13 & B24a & 10 & 43 & D48d & 1 & 73 & E96e & 8 \\
\hline 14 & $\mathrm{C} 12 \mathrm{a}$ & 59 & 44 & $\mathrm{D} 48 \mathrm{e}$ & 5 & 74 & E96f & 12 \\
\hline 15 & $\mathrm{C} 24 \mathrm{a}$ & 81 & 45 & D64a & 4 & 75 & E96g & 1 \\
\hline 16 & $\mathrm{C} 24 \mathrm{~b}$ & 109 & 46 & D64b & 2 & 76 & E96h & 2 \\
\hline 17 & $\mathrm{C} 24 \mathrm{c}$ & 109 & 47 & D64c & 70 & 77 & E96i & 2 \\
\hline 18 & $\mathrm{C} 48 \mathrm{a}$ & 224 & 48 & D64d & 4 & 78 & E96j & 2 \\
\hline 19 & D8a & 16 & 49 & D64e & 6 & 79 & E96k & 2 \\
\hline 20 & D12a & 15 & 50 & D72a & 1 & 80 & E192a & 6 \\
\hline 21 & D16a & 2 & 51 & D72b & 4 & 81 & E192b & 1 \\
\hline 22 & D16b & 3 & 52 & D72c & 4 & 82 & E192c & 4 \\
\hline 23 & D16c & 138 & 53 & D72d & 12 & 83 & E192d & 14 \\
\hline 24 & D16d & 3 & 54 & D96a & 3 & 84 & E192e & 14 \\
\hline 25 & D18a & 16 & 55 & D96b & 2 & 85 & E192f & 9 \\
\hline 26 & $\mathrm{D} 24 \mathrm{a}$ & 3 & 56 & D96c & 1 & 86 & E192g & 2 \\
\hline 27 & D24b & 3 & 57 & D96d & 2 & 87 & E192h & 4 \\
\hline 28 & $\mathrm{D} 24 \mathrm{c}$ & 5 & 58 & D128a & 4 & 88 & E192j & 2 \\
\hline 29 & D24d & 7 & 59 & $\mathrm{D} 128 \mathrm{~b}$ & 6 & 89 & E192i & 2 \\
\hline 30 & $\mathrm{D} 24 \mathrm{e}$ & 20 & 60 & $\mathrm{D} 128 \mathrm{c}$ & 6 & 90 & E384a & 2 \\
\hline № & I-G & $\mathrm{n}$ & № & I-G & $\mathrm{n}$ & № & I-G & $\mathrm{n}$ \\
\hline 91 & E384b & 2 & 109 & F96d & 1 & 127 & G192b & 1 \\
\hline 92 & E384c & 8 & 110 & F128a & 1 & 128 & G384a & 1 \\
\hline 93 & E384d & 2 & 111 & $\mathrm{~F} 128 \mathrm{~b}$ & 1 & 129 & G384b & 1 \\
\hline 94 & E768a & 4 & 112 & F144a & 2 & 130 & G384c & 1 \\
\hline 95 & E768b & 4 & 113 & F192a & 4 & 131 & G768a & 1 \\
\hline 96 & E768c & 2 & 114 & $\mathrm{~F} 192 \mathrm{~b}$ & 2 & 132 & G768b & 2 \\
\hline 97 & E1536a & 4 & 115 & F192c & 1 & 133 & G768c & 1 \\
\hline 98 & F32a & 1 & 116 & F192d & 1 & 134 & G768d & 1 \\
\hline 99 & $\mathrm{~F} 32 \mathrm{~b}$ & 1 & 117 & F192e & 1 & 135 & G1536a & 1 \\
\hline 100 & F64a & 4 & 118 & F192f & 1 & 136 & G1536b & 2 \\
\hline 101 & F64b & 1 & 119 & F256a & 6 & 137 & G1536c & 1 \\
\hline 102 & F64c & 1 & 120 & F256b & 3 & 138 & G1536d & 2 \\
\hline 103 & F64d & 3 & 121 & F256c & 3 & 139 & H192a & 1 \\
\hline 104 & F72a & 2 & 122 & F384a & 2 & 140 & $\mathrm{H} 384 \mathrm{a}$ & 1 \\
\hline 105 & $\mathrm{~F} 72 \mathrm{~b}$ & 1 & 123 & F384b & 1 & 141 & $\mathrm{H} 384 \mathrm{~b}$ & 1 \\
\hline 106 & F96a & 3 & 124 & F384c & 1 & 142 & K1536a & 1 \\
\hline 107 & F96b & 1 & 125 & G96a & 1 & & & \\
\hline 108 & F96c & 1 & 126 & G192a & 1 & & & \\
\hline
\end{tabular}

References

[1] Ю. М. Гуфран, Структруные Фазовые Переходы. c304. 1982.

\section{Volume 5 Issue 6, June 2016} www.ijsr.net

Licensed Under Creative Commons Attribution CC BY 


\section{International Journal of Science and Research (IJSR) \\ ISSN (Online): 2319-7064}

Index Copernicus Value (2013): 6.14 | Impact Factor (2015): 6.391

[2] H.T.Stokes, http://www.physics.byu.edu/ isotropy.html. 2013.

[3] C.J.Bradley, A.P.Cracknell. The Mathematical Theory of Symmetry in Solids. 1972.

[4] O. V. Kovalev, Representations of the Crystallographic Space Groups, Irreducible Representations, Induced Representations and Corepresentations. Gordon and Breach, Amstrerdam, 350. 1993.

[5] M.S. Dresselhaus et al, Group Theory Application to the Physics of Condensed Matter. Springer-Verlag Berlin Heidelberg. 2008.

[6]Л. Д. Ландау, и др. Статистическая Физика, М. Наука, 286. 2006.

[7] Kwang Son Ri, et al. Physica B: Condensed Matter. Vol. 424, 20. 2013.

Volume 5 Issue 6, June 2016 www.ijsr.net 